Intersections

Canadian Journal of Music

Revue canadienne de musique
Intersections CANADIAN JOURAL OF MUSIO

\title{
Aspects de l'ethos musical dans l'antiquité grecque
}

\section{Fabien Delouvé}

Volume 29, numéro 2, 2009

URI : https://id.erudit.org/iderudit/1000039ar

DOI : https://doi.org/10.7202/1000039ar

Aller au sommaire du numéro

\section{Éditeur(s)}

Canadian University Music Society / Société de musique des universités canadiennes

ISSN

1911-0146 (imprimé)

1918-512X (numérique)

Découvrir la revue

Citer cet article

Delouvé, F. (2009). Aspects de l'ethos musical dans l'antiquité grecque.

Intersections, 29(2), 52-65. https://doi.org/10.7202/1000039ar

\section{Résumé de l'article}

Si elles sont assez nombreuses, les études relatives à la notion d'ethos musical dans l'Antiquité souffrent du morcèlement qui les caractérise. Il en résulte que l'ethos musical est souvent ignoré car mal compris. Cet article a pour but de présenter des éléments relatifs à l'étude de cette notion pour laquelle l'absence d'intérêt des musicologues réside dans la structuration même des savoirs anciens, que l'on qualifierait aujourd'hui d'interdisciplinaire. Cette interdisciplinarité amène le chercheur à adopter une démarche épistémologique impliquant un retour aux sources premières, donc à l'étude des langues anciennes, et à exploiter des sources non musicologiques.
All Rights Reserved (C Canadian University Music Society / Société de musique des universités canadiennes, 2010
Ce document est protégé par la loi sur le droit d'auteur. L'utilisation des services d'Érudit (y compris la reproduction) est assujettie à sa politique d'utilisation que vous pouvez consulter en ligne.

https://apropos.erudit.org/fr/usagers/politique-dutilisation/ 


\title{
ASPECTS DE L'ETHOS MUSICAL DANS L'ANTIQUITÉ GRECQUE
}

\author{
Fabien Delouvé
}

S'il est une notion récurrente dans les textes antiques, c'est celle de l'ethos, associée à la musique. Cette notion dont les origines sont très anciennes, puisque l'on en retrouve des éléments dans les mythologies assyro-babyloniennes, fut largement transmise et développée tout au long de l'Antiquité. La musicologie moderne, qu'il semble courant de faire débuter au XIX ${ }^{\mathrm{e}}$ siècle avec FrançoisJoseph Fétis', a notamment traité de l'ethos musical dans l'Antiquité grecque : les écrits focalisés sur cette notion ou en traitant brièvement sont assez nombreux $^{2}$. Toutefois, il apparaît rapidement à leur lecture qu'une vision globale n'a jamais été réellement proposée, et que ceux qui l'ont fait proposèrent après recensement des textes anciens relatifs à l'ethos musical des descriptions parfois hasardeuses et erronées des notions et objets concernés. Parmi ces travaux, celui d'Hermann Abert de 1899 servit longtemps de référence sur le sujet jusqu'à l'édition de Warren Anderson de 19663. Le texte d'Abert, l'un des premiers sur la notion d'ethos musical dans l'Antiquité grecque, montre que les différents objets musicaux antiques étaient considérés comme les véhicules de l'ethos; il propose alors un recensement assez détaillé des sources relatives à cette notion, mais donne une vision assez confuse de l'articulation des nombreux éléments qui la constituent. Warren Anderson, quant à lui, a surtout développé un aspect de l'ethos: son rapport avec l'éducation, tel qu'on le trouve dans la République de Platon et les Politiques d'Aristote, ainsi que sa fonction au théâtre. Ces deux travaux méritent d'être remis à jour et complétés ; ce fut - entre autres - le but de notre thèse de doctorat ${ }^{4}$. Cet article a pour objectif de présenter l'ensemble des éléments relatifs à l'ethos musical dans l'Antiquité et de faire ainsi le point sur cette notion souvent ignorée car mal comprise. Cette ignorance et cette incompréhension résident dans la structuration même des savoirs antiques qui fonctionnent de manière interdépendante au sein de l'encyclopédies, le cercle des connaissances que tout homme libre instruit devait posséder ${ }^{6}$. Il en résulte qu'une recherche musicologique de ce type se doit d'être interdisciplinaire et de nature épistémologique. Nous présenterons donc les pistes de recherches propres à la notion musicale d'ethos: celle de la lexicologie, celle de

1 Voir par exemple Aubry 1975, p. 9, Tinel 1971, Heller 1981, p. 294, Legras 2003, p. 168.

2 Citons parmi ceux qui développent le plus cette notion: Wiegandt 1881; Walter 1890; Abert 1899 ; von Hornbostel 1929 ; Wilkinson 1938 ; Anderson 1966 ; Mathiesen 1984 ; Boccadoro 2002 ; Bundrick 2005; Woerther 2007.

3 Voir Feaver 1968.

4 Voir Delouvé 2009.

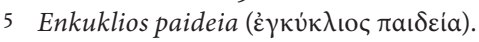

6 L'enseignement de l'encyclopédie aux hommes libres était en tout cas valable à Athènes (Flacelière 2008, p. 71 ). 
l'épistémologie conceptuelle, puis celle - mieux connue - des poètes et des philosophes.

Dans son De officiis, Cicéron dit que chaque instruction relative à un sujet entrepris par la raison doit débuter par une définition, afin que puisse être compris ce dont on parle7. Devant répondre, comme nous venons de le dire, à une démarche épistémologique, notre première étape sera donc celle de la terminologie. Il importe en effet de savoir que la première occurrence de la locution «ethos des modes » ne date que de l'Histoire et théorie de la musique de l'Antiquité, recueil de deux volumes écrits par François Auguste Gevaert (1828-1908) et édités en 1875 et $1881^{8}$. Le terme ethos ( $\left.\tilde{\eta} \theta 0 \varsigma\right)$ est, depuis Aristote, compris comme étant la partie de la rhétorique qui traite de l'impression morale produite par un orateur, des moyens qu'il doit employer pour gagner l'intérêt de l'auditoire. L'ethos se différencie du pathos ( $\pi \dot{\alpha} \theta 0 \varsigma$ ) qui soulève la passion et l'émotion c'est-à-dire les affections, les tendances, les désirs de l'auditoire sur lesquels l'orateur peut s'appuyer dans son discours persuasif 9 . L'ethos est ce vers quoi l'être humain tend naturellement. Comme l'écrit Michel Meyer dans son «Introduction » à la Rhétorique d'Aristote : « L'ethos est ce qui donne alors crédibilité au discours et le pathos, ce qui le fait accepter : les deux moments de la production et de la réception » (Meyer 1991, p. 34). Une étude approfondie de l'évolution du terme ethos ( $\tilde{\eta} \theta 0 \varsigma)$ montre son ancienneté et ses nombreuses significations : le poète à qui l'on attribue l'Iliade et l'Odyssée, Homère, l'employait sous la forme plurielle dès le $\mathrm{IX}^{\mathrm{e}}$ siècle avant notre ère en tant que " séjour habituel », « lieux familiers ", ou « demeure ", sous l'orthographe

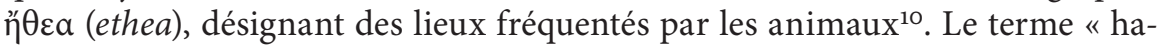
bituel » accolé à « séjour » ci-dessus comme proposition de traduction d'ethos ne doit pas être oublié : Frédérique Woerther écrit à ce sujet que chez Homère, ethos désigne avant tout un rapport au sujet, qui implique l'idée d'" habitude $»^{11}$. Les Travaux et les jours d'Hésiode proposent le substantif pluriel ethea

7 I, 3 : Placet igitur, quoniam omnis disputatio de officio futura est, ante definire, quid sit officium, quod a Panaetio praetermissum esse miror. Omnis enim, quae a ratione suscipitur de aliqua re institutio, debet a definitione proficisci, ut intellegatur, quid sit id de quo disputetur (« Il convient, puisque tout notre discours doit avoir trait à l'action moralement bonne, de la définir au préalable, ce que Panétius a négligé de faire. Toutes les fois en effet qu'on entreprend de traiter un sujet méthodiquement, on doit prendre comme point de départ une définition, afin d'avoir une idée claire de ce sujet ») le texte latin vient de Cicéron 1913 et la traduction est celle de Appuhn 1933.

8 Premier volume (1875), II, II, 202 : « Constatons d'abord qu'il est certains points par où le sentiment moderne confine sans intermédiaire au sentiment antique. Nous n'en pouvons donner une preuve plus frappante que la classification de l'éthos des modes, par rapport à leur terminaison finale sur la tonique, sur la dominante ou sur la tierce. »

9 «Aristote considérait que les preuves inhérentes au discours étaient au nombre de trois : le caractère moral de l'auditeur (l'ethos, [î̃ $\theta 0 \varsigma$ ]), la disposition de l'auditoire (le pathos [ $\pi \dot{\alpha} \theta 0 c$ ]) et le discours lui-même (le logos [ $\lambda$ ójoc]) (Aristote : Rhétorique, I, 2, III, 1356a). Il se différencierait par là des rhéteurs de son époque, selon lesquels l'ethos ne contribue pas à la persuasion » (Egges 1999, p. 31).

10 Odyssée, 14, 411; Iliade, 6, 511.

11 Ce point est la conclusion à son étude sur les dérivés d'ethos que sont aethéssein (ản $\theta \dot{\varepsilon} \sigma \sigma \varepsilon เ v$ )

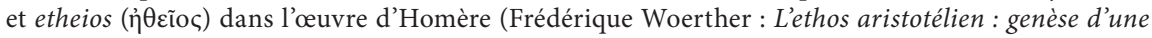
notion rhétorique, op. cit. Cette étude concerne les pp. 25-32, cette conclusion apparaissant à la p. 32. Les éléments présentés ici relatifs à l'évolution du terme ethos proviennent principalement de cet ouvrage). 
pour désigner un lieu de séjour, applicable aux êtres humains et non uniquement aux animaux comme c'était le cas chez Homère (Hésiode 1940, p. 167-168 et 220-223). Deux autres acceptions d'ethos se trouvent aussi chez Hésiode qui

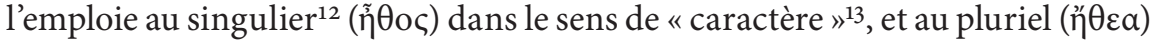
dans celui de « coutumes $»^{14}$ et $d^{\prime}$ " habitudes $»^{15}$. Le singulier $\tilde{\eta} \theta$ o c conservera ce sens d'" habitudes ", désignant alors les « mœurs » comme c'est le cas dans l'Agamemnon ${ }^{16} \mathrm{~d}^{\prime} E s c h y l e$. Pindare emploie le terme $\tilde{\eta} \theta$ os à plusieurs reprises ; une occurrence se situe dans les Odes Olympiques (XI, 20), et recouvre le sens donné par Homère de " repaire » pour les animaux, cette fois-ci au singulier $(\tilde{\eta} \theta 0 \varsigma)$; dans ces mêmes Odes (XIII, 13), il prend le sens de «fond naturel »; on rencontre aussi ce terme dans ses Odes Néméennes (VIII, 34) en tant que «manière d'être », ou "penchants ». C'est dans les Élégies ${ }^{17}$ de Théognis que ĩ $\theta$ oc est employé dans le sens de "caractère ", acception déjà rencontrée chez Hésiode. Notons que, orthographié avec un epsilon ( $\varepsilon$ [e bref]), et non avec un êta ( $\eta[e$ long]), $\check{\varepsilon} \theta$ o ( (au singulier), conserve le sens proposé par Hésiode de " coutume " dans le Philoctète ${ }^{18}$ de Sophocle et celui d'" habitudes " dans la Cyropédie ${ }^{19}$ de Xénophon. Empédocle employait le terme ñ $\theta$ oc dans le sens de "nature ", c'est-à-dire de " caractère propre ${ }^{20}$, cette traduction le rendant ici synonyme de phusis ( $\varphi v ́ \sigma ı \varsigma)$, « nature », terme qui sera employé par Aristote pour définir l'aspect éthique de certaines harmonies ${ }^{21}$, et correspondant au latin natura qui sera employé à partir du Moyen Âge dans ce sens ${ }^{22}$. Notons que l'acception de " caractère " relevée chez Hésiode et Théognis se retrouve dans des fragments attribués à Démocrite, le 57 et le 192, où « caractère » fait référence à une « disposition morale».

12 «Il s'agirait de la première apparition historique d' $\tilde{\eta} \theta$ o p. 33).

13 Les travaux et les jours, 67-68 et 78. Ceci contredit Warren Anderson qui disait que cette acception n'était valable qu'à partir d'Héraclite, Hésiode ayant vécu au moins deux siècles avant Héraclite (Anderson 1980, p. 282).

14 Vers $135-137$.

15 Les travaux et les jours, 699.

16 Vers 728. Nous contredisons ici l'affirmation du dictionnaire grec Le Grand Bailly, qui orthographie $\varepsilon$ $\theta$ o $̧$ cette occurrence (Bailly 200o, p. 581).

17 Vers 213.

18 Vers 894.

$191,6,33$.

20 Fragment 17.

21 VIII, 5, 1340a-b, section 22.

22 On trouve cette traduction par natura dans diverses éditions (Aristote 1548, p. 252), ou «Libri politicorum " (Aristote 1483, p. 564 [pagination proposée par nous-mêmes, étant inexistante dans l'édition]). Cette traduction des Politiques semble être celle de référence au Moyen Âge puisqu'on la retrouve telle quelle chez Albert le Grand (Albert Le Grand 1891, p. 803).

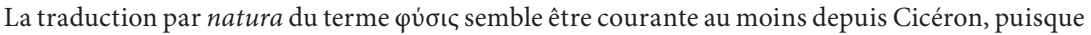
lorsqu'il traite de la notion de phusis chez Anaximandre, il traduit bien ce terme par natura : ... is enim [Anaximander] infinitatem naturae dixit esse, e qua omnia gignerentur ([Anaximandre] dit, en effet, que l'infinité de la nature est ce dont toutes choses sont engendrées) (Cicéron 1980, p. 85 ; traduction française par Marcel Conche dans Anaximandre 1991, p. 85). 
Étant donné la grande quantité d'occurrences d'ethos dans les écrits attribués à Platon ${ }^{23}$ et à Aristote ${ }^{24}$, nous laisserons ici de côté les subtilités d'emploi chez ces deux philosophes ${ }^{25}$ pour ne retenir chez eux que les acceptions ayant un rapport avec un contexte musical. L'on rencontre en effet chez Platon ce qui semble être la première application historique d'ethos à la musique, l'employant dans le sens de "dispositions morales ${ }^{26}$ dont les chants des hommes d'âge mûr et les mythes racontés par les hommes âgés sont l'expression. C'est lorsqu'Aristote présente dans ses Politiques des caractérisations " éthiques " d'harmonies qu'il applique le terme ethos à une de ces harmonies ${ }^{27}$; cet emploi unique chez Aristote, de par son attribution à un objet musical, ne se retrouve, semble-t-il, chez aucun de ses prédécesseurs et dans aucun autre passage du corpus aristotélicien. Sur les cinq occurrences que nous relevons du terme ethos dans les Éléments harmoniques d'Aristoxène de Tarente, deux sont appliquées à des objets musicaux : les genres ${ }^{28}$. Il faudra, semble-t-il, attendre les $\mathrm{II} / \mathrm{III}^{\mathrm{e}}$ siècles pour que ethos soit appliqué de manière apparemment systématique à des objets musicaux dans des écrits consacrés ou non à la musique tels le traité attribué à Plutarque ${ }^{29}$, le Banquet des Sophistes d'Athénée ${ }^{30}$, ou dans le traité de musique d'Aristide Quintilien ${ }^{31}$. Cet emploi disparaîtra d'Occident avec l'abandon progressif de la langue grecque pour le latin. Si ethos est couramment traduit en latin par mores, ce dernier terme ne sera pas retenu dans les traités lorsqu'il sera question de caractériser des objets musicaux, et notamment les huit tons, tropes ou modes du plain-chant dit " grégorien " au Moyen Âge. Les écrits nous donnent à sa place celui de natura, certainement en référence à l'emploi d'Aristote que nous avons mentionné ci-dessus, celui de proprietas se rencontrant aussi, et plus rarement celui de qualitas - surtout à partir du XIVe siècle dans certains traités.

S'intéresser aux origines du terme était une première étape. La seconde est celle des origines de la notion. L'ethos musical tire son origine dans les effets produits par la musique. Les traités de musique antiques ${ }^{32}$ ne témoignent que très rarement des sources de cette notion qui sont principalement constituées

23 Thomas Mitchell dans son Index Graecitatis Platonicae (Mitchell 1832, Tome I, p. 328-329) recensait 70 occurrences de ce terme dans l'ensemble des écrits de Platon (Gorgias, 2 occ.; République, 12 occ.; Lois, 35 occ.; Epinomis, 1 occ. ; Lettres, 10 occ. ; Lysias, 1 occ. ; Politique, 4 occ. ; Banquet, 2 occ. ; Timée, 1 occ. ; Phèdre, 2 occ.). Frédérique Woerther en dénombre 140 (Banquet, 40cc.; Cratyle, 2 occ.; Critias, 1 occ.; Gorgias, 2 occ. ; Lois, 60 occ. ; Lysis, 1 occ. ; Phédon, 1 occ. ; Phèdre, 3 occ. ; Politique, 8 occ. ; République, 33 occ. ; Timée, 1 occ. ; Lettres, 14 occ.).

24 Frédérique Woerther en dénombre 255 occurrences dans ses écrits (2007, p. 320).

25 Nous renvoyons au texte de Woerther 2007.

26 Lois, II, 664d et 669b (Platon 2006, tome 1, p. 137 et 149).

27 VIII, 12, 1342a (Aristote 1993, p. 544-5).

28 I, 23, 8-10 et II, 48, 17 (Aristoxène 1902, p. 115 et 123).

29 Sections $11,19,20,32$ et 36 .

30 XIV, 19, 624e et 20, 625b.

31 Par exemple I, 6, et II, 12.

32 Textes très peu nombreux. On peut mentionner Aristote 1993 ; les Éléments Harmoniques d'Aristoxène de Tarente (traité le plus ancien, malheureusement mutilé, parvenu jusqu'à nous [op. cit.]) ; Euclide 1884 ; Philodème 1894. Les autres traités datent des premiers siècles de notre ère : Aristide Quintilien 1999; Plutarque 1954; Ptolémée 1982 ; Nicomaque 1881 ; Sextus Empiricus 2002 ; Porphyre 1932 ; Alypius et Gaudence Alypius 1895. 
de textes cosmologiques et médicaux. Si des structurations mathématiques du cosmos sont présentées dans des textes antérieurs à la période Classique ${ }^{33}$, c'est le Timée de Platon qui propose le premier une organisation mathématicomusicale de l'Âme du Monde. Reposant sur des conceptions pythagoriciennes selon lesquelles l'ensemble des phénomènes est composé de nombres et de proportions, cette structuration de l'Âme du Monde selon des proportions numériques musicales donne les clés de la compréhension de l'essence musicale de l'âme de l'être humain qui interagit en rapport microcosmique avec l'infiniment grand. Ainsi, l'âme de l'être humain est régie par des proportions musicales. Il en résulte que la mise en action desdites proportions lors d'une représentation musicale fera vibrer par sympathie l'âme de l'auditeur qui réagira de manière positive à son audition. Inversement, l'émission d'une musique n'utilisant pas ces proportions aura un effet négatif sur l'auditeur. Si ces éléments-là ne sont pas présents dans les dialogues de Platon, ils se rencontrent dans des traités divers, tels ceux de médecine, d'architecture ou de grammatologie. Sans entrer dans des détails numériques, le traité du corpus hippocratique $D u$ Régime semble être la plus ancienne formulation écrite en langue grecque de la théorie micro-macrocosmique ${ }^{34}$. C'est dans la dixième section du premier livre de ce texte qu'est formulée une doctrine dualiste qui dit que l'être humain est constitué de deux éléments opposés et complémentaires, le feu qui est chaud et $\mathrm{sec}$, et l'eau qui est froide et humide. Et, point important pour notre propos, cette section nous dit que l'être humain est composé à l'imitation de l'Univers : les circulations du feu dans le corps sont associées à des éléments cosmiques. Les circulations vers les cavités où sont situées les humeurs " représentent la propriété de la lune », et celles qui vont vers l'extérieur " représentent la propriété des astres ${ }^{35}$. D’autres écrits issus du même corpus proposent le même type de théorie micro-macrocosmique, mais avec d'importantes différences. En ce qui concerne l'âme, Jacques Jouanna écrit qu'elle « effectue des révolutions analogues à celles du soleil, selon une orbite circulaire dont le centre est le ventre » (Jouanna 1966, p. xv). L'analogie entre la musique et la biologie découle de ce système de pensée, notamment par l'association de rythmes musicaux au rythme de la pulsation ${ }^{36}$. Toutefois, il semblerait que cette association entre le pouls et les rythmes de la prosodie ait ensuite disparu des traités de médecine pour n'être, comme l'écrit Jackie Pigeaud, qu'une « idée de lettrés " (Pigeaud 1987, p. 159).

33 Déjà attestées dans des textes paléo-babyloniens, elles sont surtout présentes chez des Présocratiques, notamment Anaximandre.

34 Voir Jouanna 1995, p. 25-66 et p. 49-50.

35 Hippocrate 1849 , p. 485-487 : "Là il (le feu) a créé trois circulations du feu arrivant l'une à l'autre en dedans et en dehors : les unes vers les cavités des humeurs, représentent la propriété de la lune ; les autres, vers la surface extérieure, vers la concrétion ambiante, représentent la propriété des

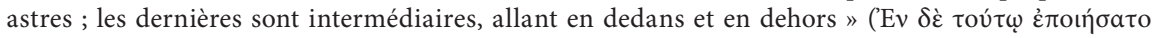

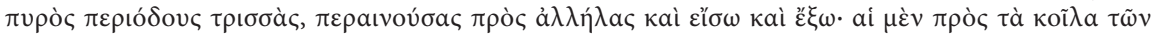

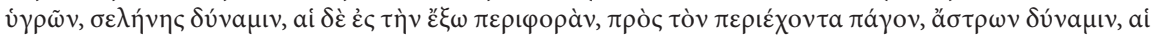

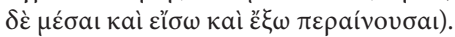

36 Voir par exemple Vitruve 1990, I, 1-15, p. 12 ; Aulu-Gelle 1967, III, 10-13, p. 167 ; Pline l'Ancien 1962, XXIX, 5, p. 21) ; et en particulier Rufus d'Éphèse 1879, p. 224-225. 
Il en résulte que la musicothérapie est l'un des effets visibles de l'association de la musique au corps humain. Si l'on se réfère à la table alphabétique du corpus hippocratique établie par Émile Littré37, cette collection de textes n'apporte aucun élément quant à un aspect curatif de la musique. Seule est présente une anecdote relatant un pouvoir négatif de l'aulos : l'affection mentale d'un certain Nicanor se déclenchait lorsqu'après avoir bu il entendait une joueuse d'aulos; cette affection se produisait uniquement la nuit, l'aulos n'ayant aucun effet sur lui le jour ${ }^{38}$. Il ne s'agit pas ici d'une guérison par la musique, mais au contraire d'une affection mentale causée par la musique, en particulier par celle de l'aulos. Ce sont en fait d'autres sources ultérieures, datées des premiers siècles de notre ère, qui font mention de l'utilisation d'une musicothérapie au cours des périodes Archaïque, Classique et Hellénistique. Plutarque ${ }^{39}$ nous dit dans son traité de musique que Terpandre guérît la peste des Lacédémoniens par la musique ${ }^{40}$. Dans sa Vie de Pythagore, Jamblique relate que le philosophe de Samos pratiquait le soir auprès de ses disciples la purification musicale des esprits agités, les aidant à trouver le sommeil, et les débarrassait au matin de leur torpeur nocturne ${ }^{41}$. Galien rapporte une réflexion de Posidonius qui, en critiquant Chrysippe, raconte une histoire où Damon d'Athènes calme par la musique de l'aulos des jeunes gens ivres rendus fous par ce même instrument ${ }^{42}$; sur les conseils de Damon, le changement du phrygien au dorien par la joueuse d'aulos calma les jeunes gens ivres. Dans l'histoire de Nicanor, l'aulos peut déclencher des affections mentales; il peut aussi énerver ou calmer dans l'anecdote rapportée par Galien. Bien que cette anecdote ne fasse pas mention d'un aspect curatif de la musique - ces jeunes gens sont seulement ivres et non pas atteints d'une maladie particulière - sa présence dans un traité attribué à Galien nous indique que certains médecins pouvaient croire à l'époque de Galien en l'influence de la musique. Athénée nous dit que Théophraste, péripatéticien de l'époque hellénistique, utilisait aussi l'harmonie phrygienne ${ }^{43}$, mais cette fois-ci pour guérir la sciatique ${ }^{44}$; Aulu-Gelle nous rapporte la même anecdote ${ }^{45}$. Cette croyance n'est pas partagée par tous les médecins, comme on peut le constater dans les Maladies chroniques du médecin Caelius Aurelianus, qui

37 Hippocrate $1839-1861$, tome 10, p. 472-848.

38 Hippocrate 1839-1861, tome 5, 81, p. 250-251.

39 Ou le Pseudo-Plutarque étant donné que la parenté de Plutarque de ce traité de musique n'est pas consensuelle chez les hellénistes.

40 Plutarque 1954, 1146c-d. p. 150.

41 Jamblique 1996, section 15, p. 65.

42 Galien 1821-1833, tome 5, p. 472-473.

43 Notons que Galien parle du phrygien (phrugion [ $\varphi \rho v ́ \gamma ı$ ov]), faisant certainement référence

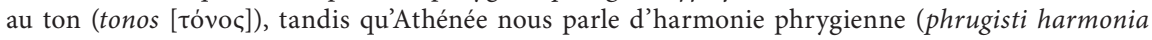

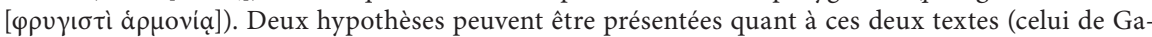
lien et celui d'Athénée). La première est que ces auteurs, non-spécialistes de musique, confondaient les tons et les harmonies, l'un nous disant que le ton phrygien excitait, l'autre que c'était l'harmonie phrygienne. La deuxième hypothèse est l'association dans ces textes des mélodies phrygiennes (ton ou harmonie) par leur pouvoir : étant donné qu'elle est phrygienne, l'harmonie du même nom aura le même pouvoir d'excitation que le ton phrygien.

44 Athénée 1854, XIV, 18, 626f, p. 995 (nous suivons ici Jackie Pigeaud qui reconnaît la sciatique en la maladie nommée par Athénée [Pigeaud 1987, p. 156]).

45 Aulu-Gelle 1967, IV, 13, p. 212. 
explique que certains médecins considèrent que des cantilènes jouées sur la $t i$ bia peuvent guérir le furor, c'est-à-dire la manie ${ }^{46}$. Caelius Aurelianus condamne cette opinion en disant que "le son de la cantilène remplit la tête", et peut même provoquer cette maladie 47 . Cette condamnation touche non seulement la tibia mais aussi un autre instrument à vent, la flûte (fistula), rejetée comme moyen de guérison de la sciatique ${ }^{48}$. Ces rejets de Caelius Aurelianus indiquent que certains médecins utilisaient la musique comme traitement de maladies de l'âme, ici la manie, et de maladies physiques, ici la sciatique. Une explication sur ces possibilités d'application de la musique à l'âme et au corps se trouve dans les Nuits attiques d'Aulu-Gelle qui pense que si la musique peut à la fois guérir les maladies de l'âme et les maladies physiques, c'est grâce à l'affinité (ou parenté49) entre l'âme et le corps par laquelle « les maladies et les remèdes de l'un et de l'autre peuvent être voisins » (Pigeaud 1987, p. 156). D'après le Timée de Platon ${ }^{50}$, cette affinité repose sur la bile, élément fondamental du rapport de l'âme et du corps, du mental et du physique (Pigeaud 1989, p. 51). Platon précise qu'il regroupe sous le nom "bile $"^{51}$ l'ensemble des humeurs ${ }^{2}$; ainsi, selon lui, ce sont les humeurs qui permettent l'affinité entre l'âme et le corps.

À côté de ces textes, que la vision dichotomique contemporaine qualifierait de «scientifiques » car traitant d'éléments propres au logos, il existe un autre corpus de sources d'effets produits par la musique : les récits des mythographes. Ces textes présentent des anecdotes bien plus spectaculaires que celles que l'on rencontre dans les écrits précédents : mythe des Sirènes, légende d'Arion, d'Amphion, d'Orphée ... Notons que ces effets spectaculaires auront leur droit de citer dans les écrits de l'Antiquité tardive, provenant d'une tradition au sein de laquelle muthos et logos semblent confondus dans certains textes de l'époque Archaïque ${ }^{53}$, tradition pourtant non dénuée de valeur historique. Cette confusion qui disparaitra avec Platon, celui-ci précisant la distinction entre muthos et logos - en particulier dans le Timée et le Parménide —, subsistera tout de même lorsqu'il sera question de présenter des effets produits par la musique, faisant que ces sources ne doivent pas être négligées.

46 La manie (mania, [ $\mu \alpha v i \alpha]$ ) est selon Jackie Pigeaud (1987, p. 7) une maladie chronique, avec altération de l'esprit sans fièvre (cette définition apportée par la médecine grecque serait difficile à dater). La manie se distingue de la phrénitis ( $\varphi \rho \varepsilon v i \tau \iota \varsigma)$ qui est une maladie aiguë accompagnée de fièvre, et se distingue de la mélancolie.

47 Caelius Aurelianus 1567, I, V, p. 308.

48 Caelius Aurelianus 1567, V, I, p. 511.

49 Adfinitas.

50 Platon 1992b, 72b-c, p. 187.

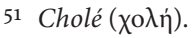

52 Platon 1992 b, 83b-c, p. 205.

53 Il y aurait même dans la pensée grecque de l'époque Archaïque une utilisation inverse de muthos et logos par rapport à l'emploi courant ultérieur (Couloubaritsis 2003, p. 58).

Notons que les historiens divisent en général l'Antiquité grecque en quatre grandes périodes : 1) La période Mycénienne de 1500 av. J.-C. À 800 av. J.-C., 2) la période Archaïque de 800 av. J.-C. À 500 av. J.-C., 3) la période Classique de 500 av. J.-C. À 350 av. J.-C., 4) la période Hellénistique ou Alexandrine (Flacelière 2008, p. 9) de 350 av. J.-C. À 30 av. J.-C., date de la disparition du royaume lagide d'Égypte (royaume Ptolémaïque) marquant l'achèvement de la conquête par Rome du Bassin Méditerranéen. 
Une fois fait le point sur le terme même qu'est ethos, et sur les origines de la notion, notre troisième étape sera celle des poètes et philosophes. Étonnamment, le terme ethos est rarement présent dans les textes anciens lors des réflexions sur l'aspect éthique de la musique ou bien lors de caractérisations d'instruments ou d'objets musicaux ; une distinction doit donc être établie entre la notion musicale d'ethos et le terme même qu'est ethos. Les poètes grecs de l'époque Archaïque appliquaient principalement des caractérisations d'ordre éthique à des mélodies et à des harmonies : Stésichore d'Himère parlait de la délicieuse découverte de la mélodie phrygienne ${ }^{54}$. Une scholie aux Péans de Pindare nous dit que «sur l'harmonie dorienne, il [Pindare] dit dans les Péans que la mélodie dorienne est la plus vénérable 55 ». Plusieurs passages des poèmes de Pindare traitent de l'influence de la musique et de son pouvoir, et attribuent un ethos à des instruments ${ }^{56}$. Les premiers vers de ses Odes Pythiques ${ }^{57}$ sont consacrés à la phorminx, et au fait que la musique de cet instrument est capable de calmer, d'endormir et d'envoûter. Des fragments attribués à Héraclite présentent des exemples relatifs au pouvoir exercé par la musique. Un de ces fragments rapporte l'extrait suivant traitant de la poétesse grecque Sapho qui aurait vécu peu de temps avant Héraclite : «Ne vois-tu pas ... quelle grâce ont les chants de Sapho qui séduisent et tiennent sous le charme ceux qui les écoutent ${ }^{258} »$ (Héraclite 1991, p. 59). Les effets de la musique ne sont ainsi pas uniquement produits par des êtres divins ou d'essence divine comme c'est le cas dans la mythologie, mais ici, par Sapho la poétesse grecque de la fin du VII siècle avant notre ère.

C'est, semble-t-il, à partir de la période Classique que l'ethos est considéré comme le fruit d'une éducation. La valeur éthique de la musique et la puissance de son influence sur le comportement humain font que Platon inscrit la mousikè avant toute autre discipline dans l'éducation de la jeunesse ${ }^{59}$. Dans la République, Platon considère que la musique et le rythme influencent l'auditeur en s'introduisant dans son âme, et s'y unissent profondément ${ }^{60}$. Ainsi, selon Platon, l'ethos est situé dans des objets mélodiques et dans des objets rythmiques. Platon dit que la musique a le pouvoir d'agir sur l'âme, et cela indépendamment de la qualité de l'exécution. Un exemple illustrant cette affirmation se trouve dans Le banquet, lorsque Alcibiade parle du satyre Marsyas : « Il

54 Poetae Lyrici Graeci 1853, fragment 34 [39], p. 749.

55 Pindare 2004, fragment n66, p. 498 pour le texte grec, et p. 499 pour le français (nous avons

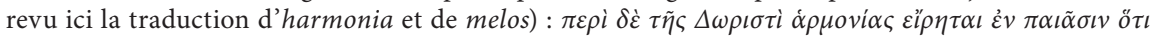

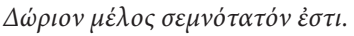

56 Voir à ce sujet l'article de Chuvin 1995, où il est question de l'importance de l'aulos dans un texte de Pindare.

57 Regroupant la première strophe et la première antistrophe.

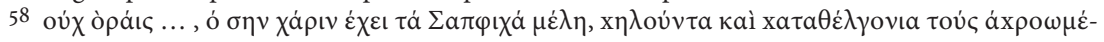
vous.

59 Platon 1993, II, 376e, p. 128. L'éducation est basée sur la mousikè et l'éducation physique

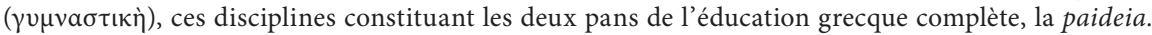
Nous pouvons constater que l'activité musicale au sein d'un chœur réunit parfaitement ces deux par-

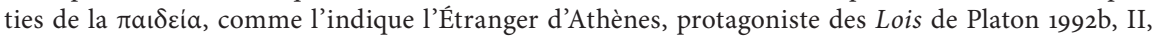
654 b, p. 117.

60 Platon 1993, III, 401d, p. 172. 
charmait les hommes par l'effet des sons que sa bouche tirait des instruments, et on les charme encore quand on joue ses mélodies [ ... ] en tout cas, qu'ils [les airs] soient joués par un grand artiste ou par une méchante joueuse d'aulos, ces airs ont seuls le pouvoir d'enchanter les cœurs ... ${ }^{61_{»}}$ (Platon 1992, 215C, p. 86)

Selon les Lois, il ne faut pas juger une mélodie d'après le plaisir, mais d'après son rapport avec l'âme ${ }^{62}$, puisque le plaisir est plutôt le résultat de l'accoutumance, et que ce sont les dieux qui font appel aux sens afin de provoquer les sentiments. Des caractérisations d'harmonies sont situées dans le troisième livre de la République. Si chaque peuple possède un caractère propre ${ }^{63}$, chaque harmonie en a également un; le nom topique de chacune d'entre elles sera à mettre en rapport avec son peuple. Platon précise dans un premier temps ${ }^{64}$ que les harmonies servant pour le thrène ${ }^{65}$ sont inutiles pour le peuple de sa cité, et qu'il faut retirer celles qui sont « relâchées » et propres aux beuveries. Il décide donc de ne conserver que deux harmonies : la dorienne et la phrygienne, puisque seules ces deux-là sont viriles, et imitent les accents de l'homme courageux contre l'adversité. De plus, elles servent en temps de paix et sont considérées comme étant utiles pour le bonheur des hommes sages. Dans la continuité des harmonies, Socrate traite ensuite des rythmes en annonçant qu'il faut «voir quels sont les rythmes d'une vie ordonnée et virile ${ }^{66}$ ». S'avouant Glaucon et lui-même comme ne connaissant pas suffisamment bien les rythmes pour pouvoir en discourir, Socrate préfère prendre conseil chez Damon d'Athènes, le maître de musique de référence que l'on retrouve nommé à plusieurs reprises dans les dialogues de Platon ${ }^{67}$. Les propos d'Aristote relatifs à la notion musicale d'ethos sont principalement situés dans le huitième livre des Politiques. Il y compare l'organisation de la cité à un chœur ${ }^{68}$ et à une harmonie ${ }^{69}$, traite de la place de la musique dans l'éducation et nous dit qu'elle fait acquérir un certain ethos $^{70}$. Dans ce huitième livre, Aristote dresse une liste des harmonies favorables à l'éducation, employant, nous le rappelons, à cet effet le terme phusis. Il y dit aussi que la musique a plusieurs utilités, servant à l'éducation (paideia), à la purification (katharsis) et au délassement (anapausis) ${ }^{71}$.

61 Nous avons préféré translittérer aulos plutôt que le traduire par «flûte » : ó $\mu \varepsilon v \gamma \varepsilon \delta$ ' ỏ $\rho \gamma a ́ v \omega \nu$

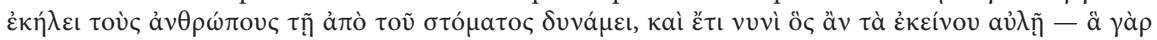

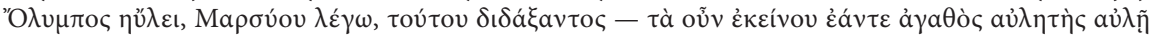

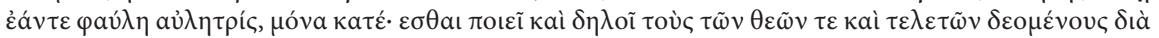

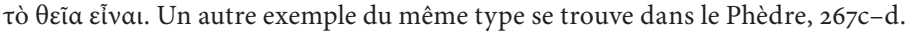

62 Platon 1992b, 655a-b, p. 119.

63 Platon 1993, IV, 435e, p. 230.

64 Platon 1993, III, 398e, p. 168.

65 Le thrène est une lamentation funèbre chantée ou psalmodiée par les femmes qui veillent le mort et l'accompagnent jusqu'à sa tombe.

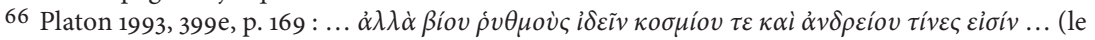
texte grec vient de Platon 1903).

67 Voir par exemple République, 40ob, et 424c (Platon 1993, p. 170 et 209); Lachès, $200 b$ (Platon 2008, p. 38).

68 Aristote 1993, III, 3, 1276b, p. 214.

69 Aristote 1993, III, 3, 1276b, p. 214. Voir aussi IV, 3, 1290a, p. 285-6.

70 Aristote 1993, VIII, 5, 1340b [24].

71 Aristote 1993, 1341b-1342a, p. 542-3. 
L’aspect mimétique de la musique se distingue chez Aristote des autres arts mimétiques parce qu'elle possède la capacité d'imiter directement les caractères (éthè $\left.{ }^{72}\right)$. Frédérique Woerther dresse en quatre points la production et la réception de la mimèsis musicale ${ }^{73}$ :

Figure 1 : La production et la réception de la mimèsis musicale

$1^{\circ}$ Les caractères (éthè) d'un homme ...

$2^{\circ}$... constituent autant de ressemblances ${ }^{74}$ dans la musique, à travers les

rythmes et les harmonies qui possèdent chacun un ethos particulier ...

$3^{\circ} \ldots$ dont l'ethos de l'âme s'imprègne lorsqu'on les écoute ...

$4^{\circ} \ldots$ pour imiter à son tour les éthè représentés par les harmonies et les

rythmes imitatifs.

En ce qui concerne les caractérisations éthiques, le huitième livre des Politiques considère que l'éducation devrait se passer des instruments de musique utilisés par les professionnels que sont la cithare et l'aulos, ceux-ci n'étant pas utiles à l'enseignement musical. Il ajoute un argument concernant le rejet de l'aulos en ce que cet instrument "n'a pas un effet éthique, mais plutôt orgiastique 75 ", apportant une caractérisation précise à un instrument de musique. Aristote considère que cet instrument devrait plutôt être utilisé dans des spectacles où il remplira une fonction purificatrice ${ }^{76}$. La place des harmonies et des rythmes dans l'éducation est le sujet du chapitre suivant des Politiques 77 . Les harmonies éthiques sont celles qui sont retenues pour l'éducation. Si Platon retenait dans la République les harmonies phrygienne et dorienne, Aristote considère que l'harmonie phrygienne " a la même vertu que l'aulos parmi les instruments. Tous deux, en effet, sont orgiastiques et passionnés ${ }^{78}$ ", et la rejette donc, conservant les mélodies doriennes qui « conviennent de préférence à l'éducation des plus jeunes ». L'harmonie lydienne est considérée comme similaire à l'harmonie dorienne, c'est-à-dire propre à l'ordre et à l'éducation. Comme nous venons de le voir pour les harmonies, les rythmes sont aussi considérés comme véhicules potentiels de l'ethos. Aristote en retient quatre, jugeant que les autres relèvent plus de la métrique pure, c'est-à-dire de la poésie, et non du langage courant : l'héroïque, l'ïambe, le trochaïque et le péan ${ }^{79}$.

Nous avons ainsi pu nous rendre compte qu'aborder l'ethos musical dans l'Antiquité était complexe. La multitude de sources à aborder est très dense, et chacune mériterait que l'on y consacre un temps d'étude approfondi et donc

72 " $\mathrm{H} \theta \eta$.

73 Woerther 2007, p. 181.

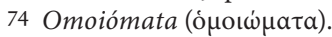

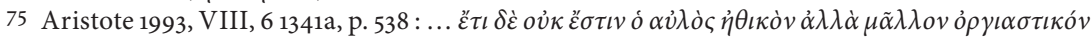
... (le texte grec vient de Aristote 1957).

76 Aristote 1993, VIII, 6 1341a, p. 538

77 Aristote 1993, VIII, 7, p. 544-5.

78 Aristote 1993, 1342b, p. 544 (le remplacement par nous-mêmes de "flûte " par " aulos " entraîne une modification des genres présents dans la traduction de Pierre Pellegrin) : $\varepsilon^{\prime} \chi \varepsilon \iota ~ \gamma \dot{\alpha} \rho \tau \dot{\eta} v \alpha \dot{v} \tau \dot{\eta} v$

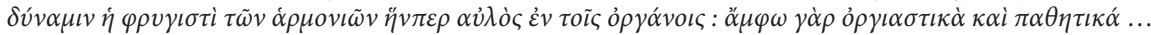

Gilbert Rouget dit que puisque l'harmonie phrygienne est qualifiée de "passionnée " (pathêtika) par Aristote, elle ne peut être douée d'ethos, mais de pathos (Rouget 1980, p. 307).

79 Rhétorique, livre III, chapitre 8, 1408b-1409a (Aristote 1991, p. 322). 
très long. De plus, nous n'avons fait ici que recenser des grandes lignes et des éléments moteurs qui devraient être exploités en profondeur pour en avoir la meilleure compréhension possible. La partie musicologique a été ici volontairement occultée : son utilité serait pourtant primordiale pour saisir de quoi parlent tous ces textes. En effet, la grande diversité d'instruments de musique caractérisés sont trop souvent peu connus; les sources antiques nous parlent de phorminx, de salpinx, d'aulos ... La multitude d'objets musicaux caractérisés mériterait aussi de longs développements que nous ne pouvons nous permettre dans le cadre de cet article : harmonies, genres, systèmes, tons, tropes ... L'aspect interdépendant des disciplines anciennes doit donc être le moyen premier de tout chercheur - incluant le musicologue - désireux de s'intéresser aux cultures antiques, comme nous venons de le constater pour l'ethos musical.

\section{RÉFÉRENCES}

Abert, Hermann. 1899. Die Lehre vom Ethos in der griechischen Musik: Ein Beitrag zur Musikästhetik des klassischen Altertums. Wiesbaden : Breitkopf \& Härtel.

Albert Le Grand. 1891. Alberti Magni Opera omnia. Paris : Auguste Borgnet.

Alypius. 1895. Alypius et Gaudence traduits en français pour la première fois. Paris : Firmin Didot.

Anaximandre. 1991. Fragments et témoignages. Paris : Presses Universitaires de France.

Anderson, Warren D. 1980. "Ethos ». The New Grove Dictionary of Music and Musicians, vol. 6, 282-287. Londres : Macmillan. .1966. Ethos and Education in Greek Music. Harvard : Harvard University Press.

Appuhn, Charles. 1933. De la vieillesse, de l'amitié, des devoirs. Paris : Garnier. Aristote. 1993. Politiques. Paris : Flammarion.

—.1993b. Problèmes. Paris : Les Belles Lettres. 1991. Rhétorique. Paris : Livre de Poche.

\section{—.1957. Aristotle's Politica. Oxford: Clarendon Press.}

-1548. Aristotelis de republica libri VIII. Paris : Michel de Vascosan.

-1483. Libri politicorum in Opera Aristotelis cum commento Averrois. Venise : Andree de Asula et Bartholomeus Alexandrinus.

Aristoxène. 1902. The Harmonics of Aristoxenus. Oxford: Clarendon Press.

Athénée. 1854. The Deipnosphists or Banquet of the Learned of Athenaeus. Londres : Henry G. Bohn.

Aubry, Pierre. 1975. La musicologie médiévale: Histoire et méthodes. Cours professé à l'Institut Catholique de Paris, 1898-1899. Genève : Slatkine Reprints. Bailly, Anatole (dir.). 200o. Le Grand Bailly. Paris : Hachette.

Boccadoro, Benno. 2002. Ethos e Varietas. Trasformazione qualitativa e modulazione nella teoria musicale dell'Antichità greca. Florence: Olschki.

Bundrick, Sheramy D. 2005. Music and Image in Classical Athens. Cambridge : Cambridge University Press. 
Caelius aurelianus. 1567. Caelii Aureliani Siccensis, medici vetusti, et in tractanda morborum curatione diligentissimi, secta methodici ( ... ) Lyon : Guillaume Rouillé.

Cicéron. 1980. Academicorum reliquiae cum Lucullo. Stuttgard : B. G. Teubner. —.1913. De Officiis, texte établi et traduit (langue anglaise) par Walter Miller. Loeb Classical Library, vol. 21. Cambridge : Harvard University Press.

Couloubaritsis, Lambros. 2003. Aux origines de la philosophie européenne - De la pensée archä̈que au néoplatonisme. Paris et Louvain : De Boeck.

Chuvin, Pierre. 1995. "Un éloge paradoxal de l'aulos dans la douzième Pythique à Midas d'Agrigente, aulète, 490 av. J.-C. ». Poésie et lyrique antiques Lille : Presses Universitaires du Septentrion.

Delouvé, Fabien. 2009. L'ethos musical de l'Antiquité à la Renaissance : Genèse, développements et mutations d'une notion. Thèse de doctorat de musicologie sous la direction de Gianfranco Vinay. Université Paris 8.

Egges, Ekkehard. 1999. "Ethos aristotélicien, conviction et pragmatique moderne ». Images de soi dans le discours - La construction de l'ethos. Lausanne et Paris : Delachaux et Niestlé S.A., 31-59.

d'Éphèse, Rufus. 1879. "Traité abrégé sur le pouls ». Euvres de Rufus d'Éphèse, texte collationné sur les manuscrits, traduit pour la première fois en français, avec une introduction, 220-232. Paris : Imprimerie Nationale, Baillière et Fils.

Euclide. 1884. L'Introduction Harmonique de Cléonide et la division du canon d'Euclide le Géomètre. Paris : Firmin Didot.

Feaver, Douglas D. 1968. "Reviewed work : Ethos and Education in Greek Music: The Evidence of Poetry and Philosophy by Warren D. Anderson ». The American Journal of Philology 89, $\mathrm{n}^{\circ} 4: 472-475$.

Flacelière, Robert. 2008. La Grèce au siècle de Périclès. Paris : Hachette Littératures, 1 ère édition 1959.

Galien. 1821-1833. Claudii Galeni opera omnia. Leipzig : Karl Knobloch.

Gevaert, François Auguste. 1895 et 1881. Histoire et théorie de la musique dans l'Antiquité, en deux tomes. Gand : Annoot-Braeckman.

Heller, Stephen. 1981. Lettres d'un musicien romantique à Paris. Paris : Flammarion.

von Hornbostel, Erich Moritz. 1929. "Tonart und Ethos ». Festschrift für Johannes Wolf zu seinem sechzigsten Geburtstag, 73-78. Berlin : Breslauer.

Héraclite. 1991. Les fragments d'Héraclite. Cognac : Fata Morgana et Roger Munier.

Hésiode. 1940. cuvres complètes. Paris : Garnier Frères.

Hippocrate. 1849. "Du régime », I, 10. Euvres complètes, tome 6, 485-487. Paris : J. B. Baillière.

Homère. 1991. Odyssée. Paris : Éditions de la Différence.

-1995. Iliade. Paris ; Arles : Actes Sud - Babel.

Jamblique. 1996. Vie de Pythagore. Paris : Les Belles Lettres.

Jouanna, Jacques. 1995. "La naissance de l'art médical occidental ». Histoire de la pensée médicale en Occident. 1. Antiquité et Moyen Âge, 25-66, 49-50. Paris : Seuil. 
1966. « La théorie de l'intelligence et de l'âme dans le traité hippocratique $D u$ régime : ses rapports avec Empédocle et le Timée de Platon». Revue des études grecques, $\mathrm{n}^{\circ} 79, \mathrm{xv}-\mathrm{xxviii}$.

Legras, Catherine. 2003. Louise Farrenc, compositrice du XIX $X^{e}$ siècle: Musique au féminin. Paris : L'Harmattan.

Mathiesen, Thomas James. 1984. "Harmonia and Ethos in Ancient Greek Music ». Journal of Musicology $\mathrm{n}^{\circ} 3$ : 264-279.

Meyer, Michel. 1991. «Introduction». Rhétorique. Paris : Livre de Poche.

Mitchell, Thomas. 1832. Index graecitatis Platonicae accedunt indices historici et geographici confecit T. Mitchell, A.M. Collegii sidneiensis apud cantabrigienses nuper socius. Oxoni e typographeo academico.

Nicomaque de Gérase. 1881. Manuel d'harmonique. Paris : Baur.

Pigeaud, Jackie. 1987. Folie et cures de la folie chez les médecins de l'Antiquité Gréco-romaine - La manie. Paris : Les Belles Lettres.

—. 1989 [1981]. La maladie de l'âme. Étude sur la relation de l'âme et du corps dans la tradition médico-philosophique antique. Paris : Les Belles Lettres.

Pindare. 2004. Euvres complètes. Paris : La Différence.

Platon. 2008. The Laches of Plato. Charleston : BiblioBazaar.

. 2006. Les lois. Paris : Flammarion.

—.1993. La République, "Du régime politique». Paris : Gallimard.

—.1992. Le banquet, Phèdre. Paris : Flammarion.

1992b. Les lois. Paris : Flammarion.

-1903. Platonis Opera. Oxford : Oxford University Press.

Pline l'Ancien. 1962. Histoire naturelle. Livre XXIX - Remèdes tirés des animaux. Paris : Les Belles Lettres.

Plutarque. 1954. De la musique. Olten \& Lausanne : Urs Graf-Verlag.

Poetae Lyrici Graeci. 1853. Londres : Williams et Norgate ; Leipzig : Beichenbach.

Porphyre. 1932. Kommentar zur Harmonielehre des Ptolemaios. Göteborg : Elander.

Ptolémée. 1982. Die Harmonielehre des Klaudios Ptolemaios. New York: Georg Olms Verlag.

Rouget, Gilbert. 1980. La musique et la transe - Esquisse d'une théorie générale des relations de la musique et de la possession. Paris : Gallimard.

Tinel, Paul. 1971. François-Joseph Fétis, père de la musicologie et polémiste. Bruxelles : Académie royale de Belgique.

Vitruve. 1990. De l'architecture. Paris : Les Belles Lettres.

Walter, Anton Friedrich. 189o. "Die ethisch-pädagogische Würdigung der Musik durch Plato und Aristoteles ». Vierteljahrschrift für Musikwissenschaft $\mathrm{n}^{\circ} 6: 388-415$.

Wiegandt, Hermann. 1881. De ethico antiquorum rhythmorum charactere auctore Aristide Quintiliano. Halle : Academia Fridericiana Halens cum Vitebergensi Consociata.

Wilkinson, Lancelot Patrick. 1938. « Philodemus on Ethos in Music ». Classical Quarterly $\mathrm{n}^{\circ} 32: 174-181$. 
Woerther, Frédérique. 2007. L’ethos aristotélicien : genèse d’une notion rhétorique. Paris : Vrin.

\title{
RÉSUMÉ
}

Si elles sont assez nombreuses, les études relatives à la notion d'ethos musical dans l'Antiquité souffrent du morcèlement qui les caractérise. Il en résulte que l'ethos musical est souvent ignoré car mal compris. Cet article a pour but de présenter des éléments relatifs à l'étude de cette notion pour laquelle l'absence d'intérêt des musicologues réside dans la structuration même des savoirs anciens, que l'on qualifierait aujourd'hui d'interdisciplinaire. Cette interdisciplinarité amène le chercheur à adopter une démarche épistémologique impliquant un retour aux sources premières, donc à l'étude des langues anciennes, et à exploiter des sources non musicologiques.

\begin{abstract}
Studies relating to the notion of musical ethos in Antiquity, while plentiful in number, have suffered from a piecemeal approach that pervades them. The result is that musical ethos is often ignored for being poorly understood. The goal of this article is to examine the discourse surrounding the study of musical ethos; it proposes that the absence of musicological interest in the subject has been caused by the structure of ancient knowledge itself, which could be characterized today as interdisciplinary. This interdisciplinary aspect leads the researcher to adopt an epistemological stance that implies a return to primary sources, and as such to the study of ancient languages and nonmusicological sources.
\end{abstract}

